\title{
Wearable Finger Pulse Oximetry for Continuous Oxygen Saturation Measurements During Daily Home Routines of Patients With Chronic Obstructive Pulmonary Disease (COPD) Over One Week: Observational Study
}

Joren Buekers ${ }^{1,2}$, MSc; Jan Theunis ${ }^{1}$, MSc; Patrick De Boever ${ }^{1,3}$, PhD; Anouk W Vaes ${ }^{4}, \mathrm{PhD}$; Maud Koopman ${ }^{4}, \mathrm{MSc}$; Eefje VM Janssen $^{4}$, MSc; Emiel FM Wouters ${ }^{4,5}, \mathrm{PhD}$; Martijn A Spruit ${ }^{4,5,6,7}$, PhD; Jean-Marie Aerts ${ }^{2}, \mathrm{PhD}$

${ }^{1}$ Health Unit, Flemish Institute for Technological Research (VITO), Mol, Belgium

${ }^{2}$ Measure, Model \& Manage Bioresponses, Department of Biosystems, KU Leuven, Leuven, Belgium

${ }^{3}$ Centre for Environmental Sciences, Hasselt University, Diepenbeek, Belgium

${ }^{4}$ Department of Research and Education, Centre of Expertise for Chronic Organ Failure (CIRO), Horn, Netherlands

${ }^{5}$ Department of Respiratory Medicine, Maastricht University Medical Centre, Maastricht, Netherlands

${ }^{6}$ Rehabilitation Research Center (REVAL), Biomedical Research Institute (BIOMED), Faculty of Rehabilitation Sciences, Hasselt University, Diepenbeek, Belgium

${ }^{7}$ School of Nutrition and Translational Research in Metabolism (NUTRIM), Maastricht University Medical Centre, Maastricht, Netherlands

\section{Corresponding Author:}

Jean-Marie Aerts, $\mathrm{PhD}$

Measure, Model \& Manage Bioresponses

Department of Biosystems

KU Leuven

Kasteelpark Arenberg 30

Leuven, 3000

Belgium

Phone: 3216321434

Email: jean-marie.aerts@kuleuven.be

\section{Abstract}

Background: Chronic obstructive pulmonary disease (COPD) patients can suffer from low blood oxygen concentrations. Peripheral blood oxygen saturation $\left(\mathrm{SpO}_{2}\right)$, as assessed by pulse oximetry, is commonly measured during the day using a spot check, or continuously during one or two nights to estimate nocturnal desaturation. Sampling at this frequency may overlook natural fluctuations in $\mathrm{SpO}_{2}$.

Objective: This study used wearable finger pulse oximeters to continuously measure $\mathrm{SpO}_{2}$ during daily home routines of COPD patients and assess natural $\mathrm{SpO}_{2}$ fluctuations.

Methods: A total of $20 \mathrm{COPD}$ patients wore a WristOx $\mathrm{x}_{2}$ pulse oximeter for 1 week to collect continuous $\mathrm{SpO}_{2}$ measurements. A SenseWear Armband simultaneously collected actigraphy measurements to provide contextual information. $\mathrm{SpO}_{2}$ time series were preprocessed and data quality was assessed afterward. Mean $\mathrm{SpO}_{2}, \mathrm{SpO}_{2} \mathrm{SD}$, and cumulative time spent with $\mathrm{SpO}$ below $90 \%$ (CT90) were calculated for every (1) day, (2) day in rest, and (3) night to assess $\mathrm{SpO}_{2}$ fluctuations.

Results: A high percentage of valid $\mathrm{SpO}_{2}$ data (daytime: 93.27\%; nocturnal: 99.31\%) could be obtained during a 7-day monitoring period, except during moderate-to-vigorous physical activity (MVPA) (67.86\%). Mean nocturnal $\mathrm{SpO}_{2}(89.9 \%$, SD 3.4) was lower than mean daytime $\mathrm{SpO}_{2}$ in rest $(92.1 \%$, SD 2.9; $P<.001)$. On average, $\mathrm{SpO}_{2}$ in rest ranged over 10.8\% (SD 4.4) within one day. Highly varying CT90 values between different nights led to 50\% (10/20) of the included patients changing categories between desaturator and nondesaturator over the course of 1 week.

Conclusions: Continuous $\mathrm{SpO}_{2}$ measurements with wearable finger pulse oximeters identified significant $\mathrm{SpO}_{2}$ fluctuations between and within multiple days and nights of patients with COPD. Continuous $\mathrm{SpO}_{2}$ measurements during daily home routines of patients with COPD generally had high amounts of valid data, except for motion artifacts during MVPA. The identified 
fluctuations can have implications for telemonitoring applications that are based on daily $\mathrm{SpO}_{2}$ spot checks. CT90 values can vary greatly from night to night in patients with a nocturnal mean $\mathrm{SpO}_{2}$ around $90 \%$, indicating that these patients cannot be consistently categorized as desaturators or nondesaturators. We recommend using wearable sensors for continuous $\mathrm{SpO}_{2}$ measurements over longer time periods to determine the clinical relevance of the identified $\mathrm{SpO}_{2}$ fluctuations.

(JMIR Mhealth Uhealth 2019;7(6):e12866) doi: $\underline{10.2196 / 12866}$

\section{KEYWORDS}

COPD; oxygen saturation; finger pulse oximeter; wearable sensor; nocturnal desaturation; telemonitoring

\section{Introduction}

Chronic obstructive pulmonary disease (COPD) is a highly prevalent lung disease that is characterized by persistent airflow limitation due to a mixture of obstructive bronchiolitis and emphysema [1]. Morbidity and mortality of COPD are high and still increasing [2], leading COPD to become the third-leading cause of death worldwide by 2030 [3]. COPD patients can suffer from low blood oxygen concentrations due to gas exchange abnormalities [1]. Hypoxemia during the night (ie, nocturnal desaturation) is also common in patients with COPD [4-6] due to nocturnal alveolar hypoventilation and ventilation-perfusion mismatching [7]. Hypoxemia can worsen with increasing disease severity [8]. Furthermore, it has been shown that hypoxemia is associated with lower exercise tolerance; decreased quality of life; increased risk for exacerbations, defined as "a sustained worsening of the patient's condition, from the stable state and beyond normal day-to-day variations" [9]; and higher risks of death [8].

The presence of hypoxemia can be assessed by invasive blood gas analyses. A noninvasive method to assess peripheral blood oxygen saturation $\left(\mathrm{SpO}_{2}\right)$ is pulse oximetry. Spot check $\mathrm{SpO}_{2}$ measurements with thresholds of $88 \%-92 \%$ have been suggested for the detection of hypoxemia $[1,10,11]$. In telemonitoring applications, daily $\mathrm{SpO}_{2}$ spot checks are used to raise alerts for exacerbations when $\mathrm{SpO}_{2}$ spot check values drop below predefined $\mathrm{SpO}_{2}$ thresholds [12]. Nocturnal desaturation is usually defined as having an $\mathrm{SpO}_{2}$ value below $90 \%$ for more than $30 \%$ of the time in bed, measured during one $[6,13-16]$ or two nights [5,17-20].

The current $\mathrm{SpO}_{2}$ monitoring strategies do not take into account natural fluctuations in $\mathrm{SpO}_{2}$. Sampling $\mathrm{SpO}_{2}$ with such a low time frequency or during such a short time period may thus lead to classification errors (ie, hypoxemic or nonhypoxemic and nocturnal desaturator or nondesaturator) or false alerts in telemonitoring applications [12]. Wearable finger pulse oximeters provide the possibility to collect $\mathrm{SpO}_{2}$ data at higher time frequencies and over longer time periods. This makes it possible to assess and account for oxygen saturation fluctuations in patients with COPD. Therefore, the objective of this study was to use wearable finger pulse oximeters to continuously measure $\mathrm{SpO}_{2}$ during daily home routines of COPD patients and assess natural $\mathrm{SpO}_{2}$ fluctuations. We hypothesized that significant natural $\mathrm{SpO}_{2}$ fluctuations are present within and between multiple days and nights, which may lead to classification errors (ie, nocturnal desaturator or nondesaturator) or false alerts in telemonitoring applications.

\section{Methods}

\section{Study Design and Participants}

Figure 1 provides a general overview of the methods applied in this single-center, 1-week observational study. COPD patients at Global Initiative for Chronic Obstructive Lung Disease stages II-IV (GOLD II-IV) were recruited at the Centre of Expertise for Chronic Organ Failure (CIRO), a COPD treatment center located in Horn, the Netherlands, during a standard baseline assessment prior to pulmonary rehabilitation [21]. The target sample size was set a priori to 20 patients. COPD patients were eligible to enroll in the study based on the following criteria: (1) clinically stable (ie, no exacerbation in the past 4 weeks), (2) no rollator use, and (3) no long-term oxygen therapy. Patients that were interested in participating were called a few days after the baseline assessment to schedule a home visit for the delivery of the wearable sensors. During this visit, the functioning of the sensors and data acquisition protocol were explained, written informed consent was obtained, and a new visit was planned for collection of the sensors at the end of the study period. During the 7-day study period, which took place before the start of pulmonary rehabilitation, two phone calls were made to resolve potential technical difficulties. Demographics, oxygen partial pressure in arterial blood $\left(\mathrm{PaO}_{2}\right)$, postbronchodilator pulmonary function data (ie, forced expiratory volume in 1 second [FEV1], forced vital capacity [FVC], and transfer factor for carbon monoxide [TLCO]), 6-minute walking distance (6MWD), and COPD assessment test (CAT) results were collected during the standard baseline assessment at CIRO. The study was approved by the Medical Research Ethics Committees United (MEC-U) (study approval number: NL58079.100.16) in the Netherlands and executed between December 2016 and April 2018. 
Figure 1. General overview of the applied methods. COPD: chronic obstructive pulmonary disease; $\mathrm{SpO}_{2}$ : peripheral blood oxygen saturation; CT90: cumulative time spent with $\mathrm{SpO}_{2}$ below $90 \%$.

1 week at home

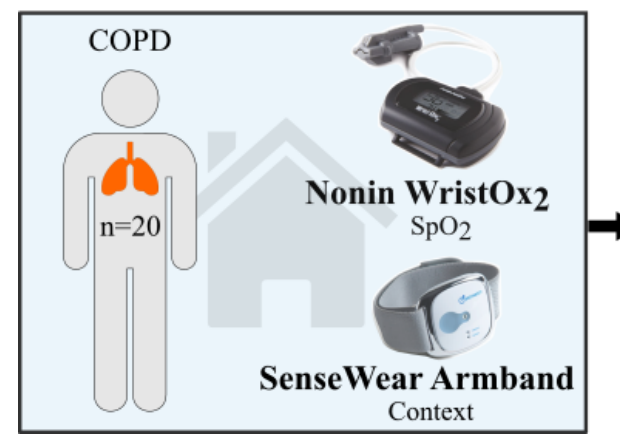

\section{Wearable Sensors and Protocol}

Continuous $\mathrm{SpO}_{2}$ measurements were performed for 1 week using a wearable finger pulse oximeter: WristOx 23150 (Nonin Medical). Nonin oximeters have frequently been used for home monitoring of patients with COPD [12] and the WristOx $x_{2} 3150$ model complies with the International Organization for Standardization (ISO) standards ISO 10993-1 and ISO 80601-2-61. The manufacturer reports an accuracy of $\pm 2 \%$ for $\mathrm{SpO}_{2}$ measurements [22]. Sampling frequency was fixed at 1 $\mathrm{Hz}$. Only the cumulative measurement time was visible for the participants. $\mathrm{SpO}_{2}$ values were not shown on the WristOx 2 display to prevent patients from changing their behavior when deviating $\mathrm{SpO}_{2}$ values would occur. Every participant received three WristOx $x_{2}$ devices to deal with the limited battery life (ie, 48 hours of continuous measurements with one WristOx ${ }_{2}$ ). Participants were instructed to wear the WristOx ${ }_{2}$ on the index finger of their nondominant hand every night and as much as possible during the day, depending on their daily routines and comfort of wearing the finger clip. Raw data were stored on the internal memory of the WristOx $x_{2}$ and downloaded at the end of the week using nVISION software, version 6.4.0.10 (Nonin Medical).

Simultaneously, actigraphy was performed with the SenseWear Armband (BodyMedia) for obtaining contextual information about physical activity levels and when the participants were lying down and/or asleep. The SenseWear Armband is a multisensory triaxial accelerometer, combining accelerometry with measurements of heat flux, galvanic skin response, and skin temperature. Based on these measurements, the armband provides information about, for example, energy expenditure (EE), expressed as metabolic equivalent of task (MET), or steps taken, while also indicating when the wearer is lying down and sleeping, at a standard sampling time of 1 sample per minute. Measurements of MET were used for classifying physical activity levels, whereas the indications of lying down and sleeping were used to separate daytime measurements from nocturnal measurements, as further described below. The SenseWear Armband has been shown to be accurate for measurements of both physical activity [23,24], except when using a rollator [24], and sleep estimations [25]. Patients were asked to wear the armband on the left upper arm, except when there was contact with water (eg, when taking a shower). Battery life of one SenseWear Armband was sufficient for continuous 24-hour measurements with a 1-minute sampling time during the whole week. Data were stored on the internal memory and downloaded at the end of the week using the BodyMedia SenseWear 8.1 software (BodyMedia).

\section{Data Preprocessing}

Figure 2 visualizes the preprocessing steps. The SenseWear Armband indications about lying down and sleep were used to determine the time of going to bed in the evening and the time of getting out of bed in the morning. These time stamps were used to divide $\mathrm{SpO}_{2}$ data into nocturnal and daytime data (see Figure 2A). Only full-night nocturnal $\mathrm{SpO}_{2}$ measurements were retained for further analyses and daytime $\mathrm{SpO}_{2}$ measurements were retained if there was at least one hour of $\mathrm{SpO}_{2}$ measurements during that day. One hour of daytime measurements was considered sufficient to examine whether significant fluctuations occurred in $\mathrm{SpO}_{2}$ values during the day. Previous studies examining intraday fluctuations only examined measurements of one hour or less [26-29]. No days or nights had to be excluded due to lacking SenseWear Armband measurements. Days with at least one hour of measurements in both the afternoon and evening were used for the comparison between afternoon (ie, 13:00-18:00) and evening (ie, 18:00-going to bed) $\mathrm{SpO}_{2}$ values. No comparison was performed between $\mathrm{SpO}_{2}$ values in the morning (ie, before 13:00) and the afternoon and evening, because $\mathrm{SpO}_{2}$ measurements were often not performed before 13:00 (see Multimedia Appendix 1). 
Figure 2. Visualization of the different preprocessing steps. Panel A shows all original data, containing error values, that are divided into daytime and nocturnal data. Panel B zooms in on the effect of data exclusion on a specific part of daytime peripheral blood oxygen saturation (SpO $\left.\mathrm{S}_{2}\right)$ data. Panel $\mathrm{C}$ zooms in on the effect of down-sampling and interpolating on the same part of daytime $\mathrm{SpO}_{2}$ data.
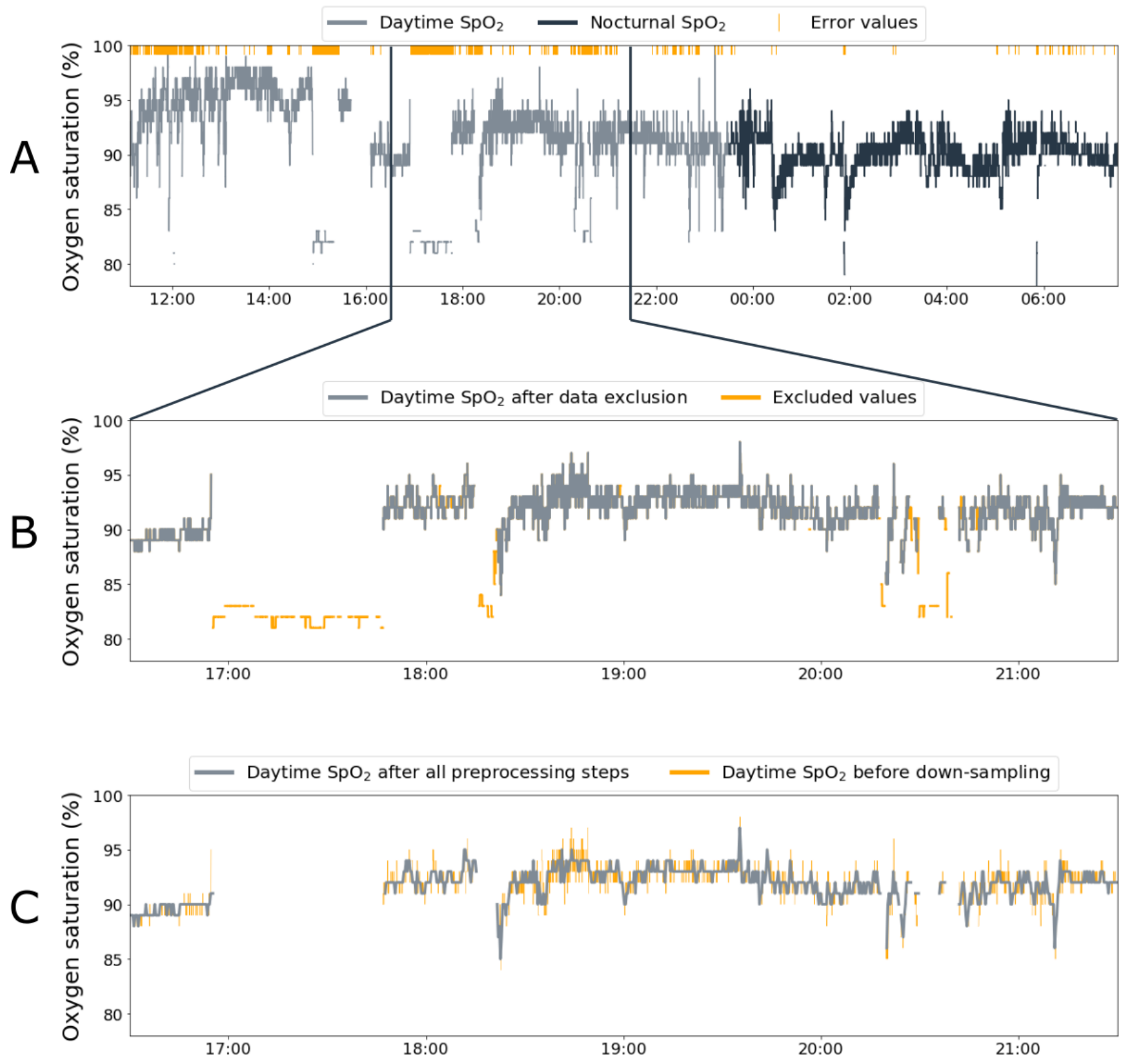

The raw $\mathrm{SpO}_{2}$ data contained error values (ie, the number 500 was provided in the raw data file) when measurements were considered invalid by the algorithms of the WristOx manufacturer (ie, orange dashes in Figure 2A). However, close inspection of the time series indicated that invalid data (eg, sudden low values) surrounded these error values. A data-cleaning algorithm was developed to exclude these invalid $\mathrm{SpO}_{2}$ values as follows. First, small blocks of data (ie, less than 20 samples) between error values were excluded. Second, bigger blocks of data (ie, between 20 and 100 samples) between error values were excluded, only when the mean $\mathrm{SpO}_{2}$ value of this data block was deviating more than $6 \%$ from the mean $\mathrm{SpO}_{2}$ value of the full day or night under consideration: both steps are shown in Figure 2B. Thorough visual and raw data examination of both valid and invalid data led to the choice of this $6 \%$ threshold for excluding invalid blocks of data (eg, Figure
2B: around 17:00-18:00). Third, remaining outliers were excluded by down-sampling the data (see Figure 2C). Autocorrelation analyses indicated that a sampling time of 20 seconds was appropriate (see Multimedia Appendix 2) and all data were thus down-sampled by taking the median $\mathrm{SpO}_{2}$ value of each consecutive 20-second block. By taking the median value, the effect of outliers was excluded. As a last step, small gaps of $\mathrm{SpO}_{2}$ data (ie, 3 or fewer missing samples) were filled using linear interpolation (see Figure 2C). After application of the data-cleaning algorithm, all data were visually checked to ensure valid $\mathrm{SpO}_{2}$ values were retained, while invalid values were removed.

\section{Data Analyses}

The SenseWear Armband indications about lying down and sleep were used to calculate the total night sleeping time (TNST) (ie, sum of all minutes indicated as sleep), wake time after sleep 
onset (WASO) (ie, sum of all minutes spent awake during the time in bed, after the first onset of sleep), and sleep efficiency (Seff) (ie, the ratio between TNST and time in bed) for every separate night. Weekly averages of TNST, WASO, and Seff were calculated to describe the sleep quality of the included patients.

Daytime $\mathrm{SpO}_{2}$ data were divided into daytime data during rest (EE $\leq 1.5$ MET, while the patient was still awake), during low-intensity physical activities (LIPAs) (1.5 MET<EE $\leq 3$ MET), and during moderate-to-vigorous intensity physical activities (MVPAs) (EE>3 MET). Data quality of the continuous $\mathrm{SpO}_{2}$ measurements was assessed for both nocturnal and daytime data based on the amount of valid data (ie, excluding error values and cleaned values). Furthermore, the effect of physical activity on the amount of valid data was examined by comparing data quality during MVPA with data quality during rest and LIPA (EE $\leq 3 \mathrm{MET})$.

Mean $\mathrm{SpO}_{2}, \mathrm{SpO}_{2} \mathrm{SD}$, and cumulative time spent with $\mathrm{SpO}_{2}$ below $90 \%$ (CT90) were calculated for every separate (1) day, (2) day in rest, and (3) night. Hereafter, the weekly average and weekly range (ie, the difference between the maximum and minimum value over the different days or nights of the same patient) of these features were calculated for every patient.

Intraday and intranight fluctuations were quantified as the weekly average of the standard deviation of the $\mathrm{SpO}_{2}$ measurements. The range of $\mathrm{SpO}_{2}$ values during the day in rest, calculated as the difference between the maximum and minimum $\mathrm{SpO}_{2}$ values of that day in rest, was determined to indicate how much spot-check values in telemonitoring applications could differ depending on the moment of the measurement. Only daytime $\mathrm{SpO}_{2}$ in rest was considered because only at these moments could spot checks have been performed in a telemonitoring application. In addition, the difference between mean $\mathrm{SpO}_{2}$ values in rest in the afternoon (ie, between 13:00 and 18:00) and in the evening (ie, between 18:00 and going to bed) was calculated for every day separately to examine differences in $\mathrm{SpO}_{2}$ baseline levels during the day.

Night-to-night and day-to-day $\mathrm{SpO}_{2}$ fluctuations were quantified as the weekly ranges of the three features (ie, mean $\mathrm{SpO}_{2}, \mathrm{SpO}_{2}$ $\mathrm{SD}$, and CT90). Furthermore, we examined how many of the included patients changed category between nocturnal desaturator and nondesaturator.

\section{Statistical Analysis}

Patient characteristics, weekly averages, and weekly ranges were summarized for all patients as mean and SD. Paired-sample $t$ tests were used to test for differences between weekly averages of nocturnal and daytime $\mathrm{SpO}_{2}$ in rest. Pearson correlations assessed the relationship between mean $\mathrm{SpO}_{2}$ and patient characteristics, intraday $\mathrm{SpO}_{2}$ fluctuations, or intranight $\mathrm{SpO}_{2}$ fluctuations. A $P$ value of $<.05$ was considered statistically significant. All analyses were carried out in Jupyter Notebooks (Project Jupyter) [30] using the Python 3.5 programming language (Python Software Foundation) [31].

\section{Results}

\section{Participants}

A total of 21 out of 41 patients that were approached accepted study participation. One patient suffered from an exacerbation before the start of the 7-day study period and was excluded. This finally led to the inclusion of 20 patients ( 14 males, $70 \%$; 6 females, $30 \%)$ with moderate $(8 / 20,40 \%)$, severe $(10 / 20$, $50 \%)$, or very severe $(2 / 20,10 \%)$ COPD. General demographics, postbronchodilator lung function, resting arterial blood gases, 6MWD, CAT score, and sleep-quality characteristics are summarized in Table 1. 
Table 1. Characteristics of the 20 included patients with moderate-to-very severe COPD ${ }^{\mathrm{a}}$.

\begin{tabular}{ll}
\hline Characteristics & Mean (SD) \\
\hline Age (years) & $63(8)$ \\
Body mass index $\left(\mathrm{kg} / \mathrm{m}^{2}\right)$ & $26(4)$ \\
Forced expiratory volume in 1 second (L) & $1.4(0.5)$ \\
Forced expiratory volume in 1 second (\% predicted) & $48(15)$ \\
Forced vital capacity (L) & $3.8(1.1)$ \\
Forced vital capacity $(\%$ predicted) & $97(20)$ \\
Transfer factor for carbon monoxide (\% predicted) & $50(16)$ \\
Partial pressure of oxygen (kPa) & $8.7(1.7)$ \\
Partial pressure of carbon dioxide $(\mathrm{kPa})$ & $5.2(0.6)$ \\
6-minute walking distance (m) & $411(59)$ \\
COPD assessment test score & $21(5)$ \\
Total night sleeping time (min) & $411(72)$ \\
Wake time after sleep onset (min) & $74(36)$ \\
Sleep efficiency (\%) & $83(8)$ \\
\hline
\end{tabular}

${ }^{\mathrm{a} C O P D}$ : chronic obstructive pulmonary disease.

\section{Continuous $\mathrm{SpO}_{2}$ Measurements, Preprocessing, and Data Quality}

An overview of the amount of $\mathrm{SpO}_{2}$ measurements that were included for further analyses is provided in Table 2 and Multimedia Appendix 1. A total of 2 days and 2 nights of measurements from patient 4 were missing due to battery issues. A total of 3 days and 3 nights of measurements from patient 11 were excluded because correct time stamps were missing, due to the patient unintentionally resetting the time indication by taking out the batteries. The last day and night of patient 11's measurements could not be analyzed because no clear distinction could be made between daytime and nocturnal data. Still, 3 days and 3 nights of patient 11's measurements were used for further analyses.

The $\mathrm{SpO}_{2}$ dataset contained $1.83 \%$ error values and an additional $1.49 \%$ were excluded during the first two steps of the data-cleaning algorithm (see Figure 3), resulting in $96.68 \%$ of valid data. Nocturnal data had $99.31 \%$ of valid data $(0.45 \%$ error data and $0.24 \%$ cleaned) compared to $93.27 \%$ of valid data during the day (3.62\% error data and $3.11 \%$ cleaned). This was similar when only considering daytime data during rest and LIPA (EE $\leq 3$ MET; $93.90 \%$ of valid data: $3.25 \%$ error data and $2.85 \%$ cleaned). However, during MVPA (EE $>3$ MET), the amount of valid data decreased to $67.86 \%$ (18.34\% error data and $13.80 \%$ cleaned). 
Table 2. Summary of the amount of peripheral blood oxygen saturation $\left(\mathrm{SpO}_{2}\right)$ measurements that were included for further analyses ${ }^{\mathrm{a}}$.

\begin{tabular}{|c|c|}
\hline Measurements included for further analyses & Mean (SD) \\
\hline Days per patient & $5.3(1.8)$ \\
\hline Nights per patient & $5.9(1.2)$ \\
\hline \multicolumn{2}{|l|}{ Hours per day } \\
\hline Total & $7.8(3.9)$ \\
\hline During rest $\left(\mathrm{EE}^{\mathrm{b}} \leq 1.5 \mathrm{MET}^{\mathrm{c}}\right)$ & $6.1(3.1)$ \\
\hline During LIPA ${ }^{\mathrm{d}}(1.5 \mathrm{MET}<\mathrm{EE} \leq 3 \mathrm{MET})$ & $1.5(2.7)$ \\
\hline During $\operatorname{MVPA}^{\mathrm{e}}(\mathrm{EE}>3 \mathrm{MET})$ & $0.2(0.2)$ \\
\hline Hours per night & $8.0(0.9)$ \\
\hline \multicolumn{2}{|l|}{ Data samples per patient } \\
\hline Total & $296,533(95,580)$ \\
\hline During the day & $149,665(88,909)$ \\
\hline During the night & $169,318(35,488)$ \\
\hline
\end{tabular}

\footnotetext{
${ }^{\mathrm{a}}$ All 20 patients performed nocturnal measurements and 17 patients performed daytime measurements. Therefore, averages were calculated with 17 patients for daytime measurements and 20 patients for nocturnal and total measurements.

${ }^{\mathrm{b}} \mathrm{EE}$ : energy expenditure.

${ }^{\mathrm{c}}$ MET: metabolic equivalent of task.

${ }^{\mathrm{d}}$ LIPA: low-intensity physical activity.

${ }^{\text {e }}$ MVPA: moderate-to-vigorous physical activity.
}

Figure 3. The amount of invalid data of continuous peripheral blood oxygen saturation $\left(\mathrm{SpO}_{2}\right)$ measurements for different activities. LIPA: low-intensity physical activity; MVPA: moderate-to-vigorous physical activity.

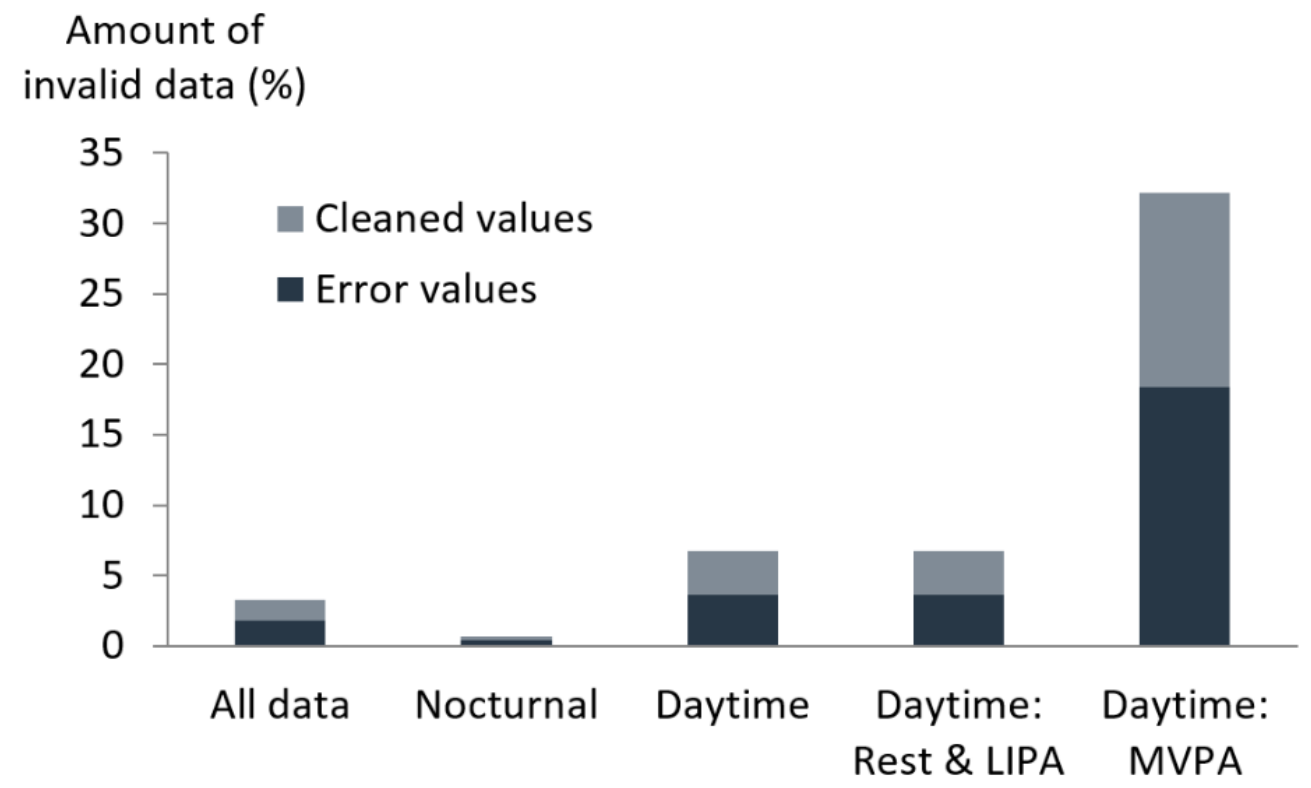

\section{$\mathrm{SpO}_{2}$ Features and Fluctuations}

The weekly averages of mean $\mathrm{SpO}_{2}$ were $89.9 \%$ (SD 3.4), $91.9 \%$ (SD 3.1), and $92.1 \%$ (SD 2.9) for nocturnal $(n=20)$, daytime $(\mathrm{n}=17)$, and daytime-in-rest $(\mathrm{n}=17)$ measurements, respectively. Figure 4 shows that mean nocturnal $\mathrm{SpO}_{2}$ was lower than mean daytime $\mathrm{SpO}_{2}$ in rest $(P<.001)$. Weekly averages of mean $\mathrm{SpO}_{2}$ were between $84.7 \%$ and $96.0 \%$ for daytime $\mathrm{SpO}_{2}$ in rest and between $80.3 \%$ and $94.3 \%$ for nocturnal $\mathrm{SpO}_{2}$ (see Figure 4).
The weekly averages of CT90 were 35\% (SD 34), 20\% (SD 29 ), and $18 \%$ (SD 29) for nocturnal, daytime, and daytime-in-rest measurements, respectively. No significant correlations were found between patient characteristics and weekly averages of the $\mathrm{SpO}_{2}$ features.

Intraday and intranight $\mathrm{SpO}_{2}$ fluctuations were quantified as the weekly average of $\mathrm{SpO}_{2} \mathrm{SD}$ for nocturnal (1.6\%, SD 0.6), daytime (1.8\%, SD 0.7), and daytime-in-rest (1.6\%, SD 0.6) measurements. Nocturnal, daytime, and daytime-in-rest $\mathrm{SpO}_{2}$ 
$\mathrm{SD}$ values were inversely correlated with nocturnal, daytime, and daytime-in-rest mean $\mathrm{SpO}_{2}$ values (all: $P<.001, \mathrm{R}^{2}>0.4$ ), respectively. On average, $\mathrm{SpO}_{2}$ in rest values ranged over $10.8 \%$ (SD 4.4) within one day. There was a significant difference between mean $\mathrm{SpO}_{2}$ in the afternoon and in the evening $(P<.001)$ (see Multimedia Appendix 3), with an average difference of $0.8 \%$ (SD 0.7) for those days where patients performed at least one hour of measurements during both the afternoon and evening. For this comparison, a total of 46 days from 12 different patients were used (median of 4 days per patient).

Night-to-night and day-to-day $\mathrm{SpO}_{2}$ fluctuations were quantified as the weekly ranges of mean $\mathrm{SpO}_{2}(2.0 \%$, SD $1.1 ; 1.9 \%, \mathrm{SD}$ $1.5 ; 1.9 \%$, SD 1.5$)$ and CT90 (28\%, SD $25 ; 19 \%$, SD $20 ; 18 \%$,
SD 21) for nocturnal, daytime, and daytime-in-rest measurements, respectively. A more detailed analysis of the night-to-night changes in CT90 is shown in Figure 5. When considering the definition of a nocturnal desaturator, three types of patients can be distinguished: consistent desaturators $(5 / 20$, $25 \%)$, consistent nondesaturators $(5 / 20,25 \%)$, and occasional desaturators that changed category over the 7 nights $(10 / 20$, $50 \%)$. A total of 6 occasional desaturators $(6 / 10,60 \%)$ were desaturators or nondesaturators in the first two nights, but changed category when $\mathrm{SpO}_{2}$ measurements of another night were considered. The weekly average of mean $\mathrm{SpO}_{2}$ for occasional desaturators was between $89.5 \%$ and $91.9 \%$, compared to $80.3 \%-88.8 \%$ and $91.8 \%-94.3 \%$ for consistent desaturators and consistent nondesaturators, respectively. 
Figure 4. Mean nocturnal peripheral blood oxygen saturation $\left(\mathrm{SpO}_{2}\right)$ compared to mean daytime $\mathrm{SpO}_{2}$ in rest. The dots indicate weekly averages of mean $\mathrm{SpO}_{2}$ for every patient, lines indicate the standard deviation of the mean $\mathrm{SpO}_{2}$ values over different days and nights, and the orange line is the line of equality.

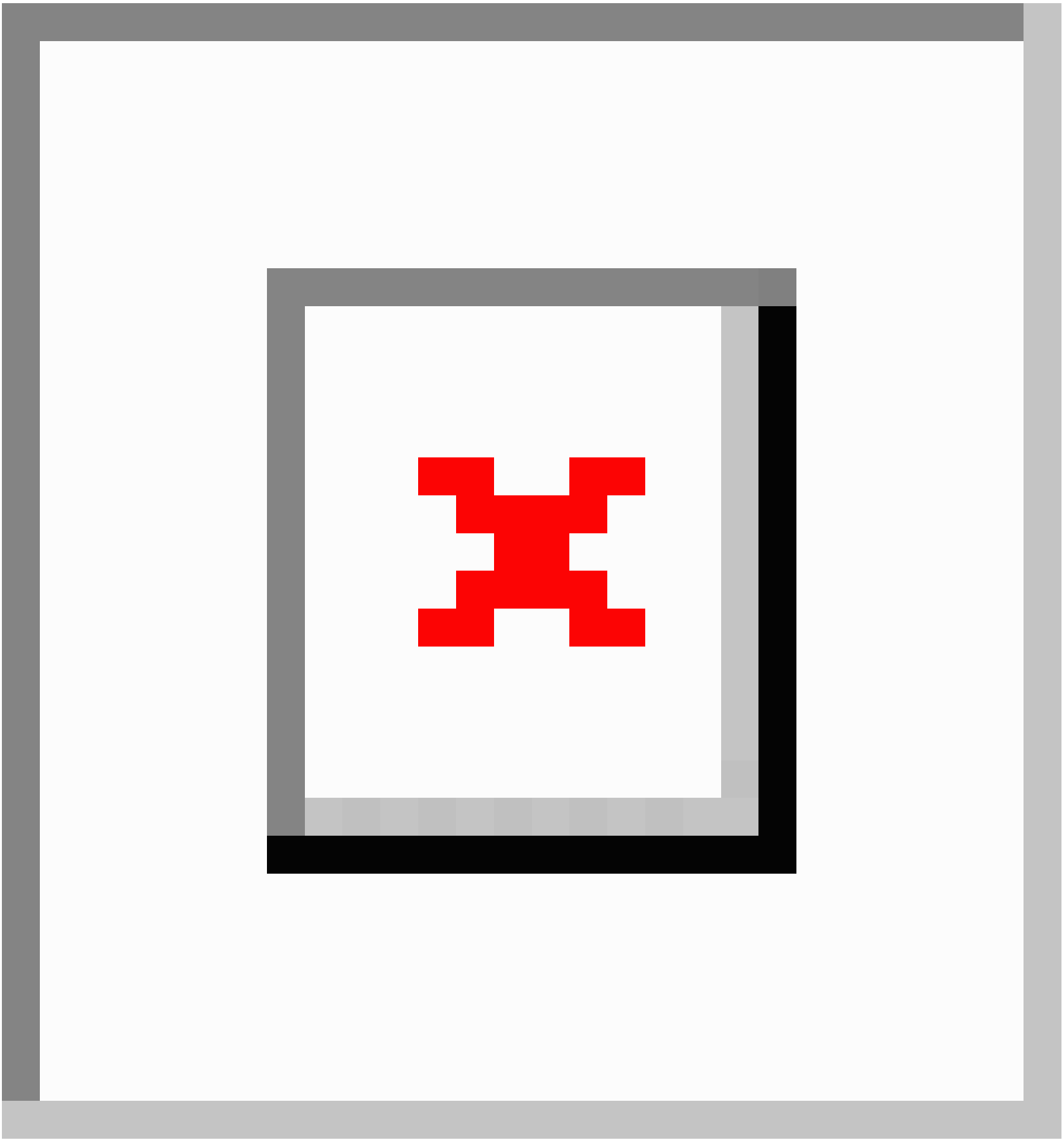


Figure 5. Cumulative time spent with peripheral blood oxygen saturation $\left(\mathrm{SpO}_{2}\right)$ below $90 \%$ (CT90) values for every night of every patient showed that $50 \%(10 / 20)$ of the included patients changed category between desaturator and nondesaturator. The number next to each dot indicates the corresponding night of the measuring week and the orange line indicates the threshold of CT90 (30\%) that divides nights with and without desaturation. A missing night number indicates that no data was available for that night.

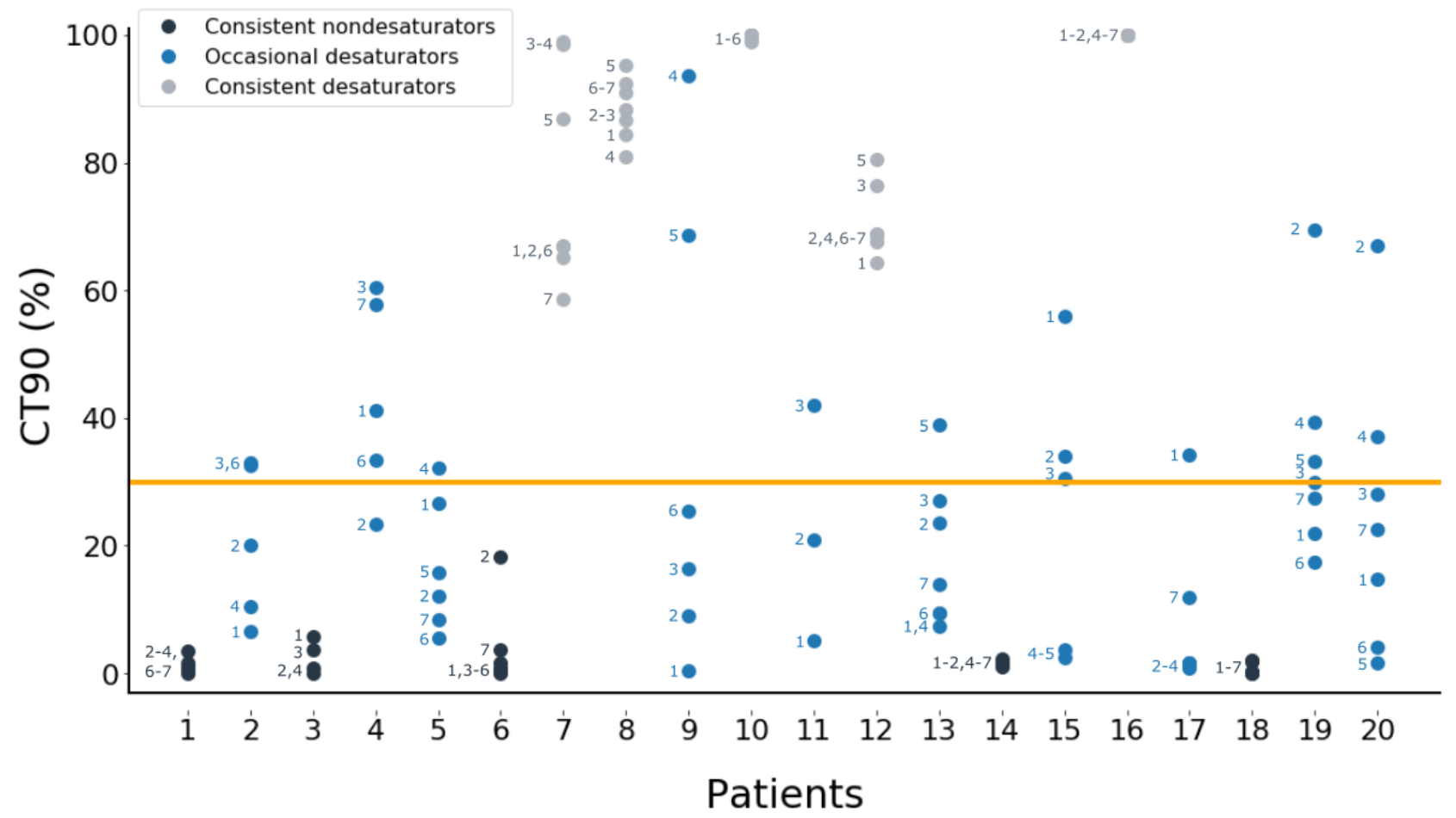

\section{Discussion}

Continuous $\mathrm{SpO}_{2}$ measurements with wearable pulse oximeters identified significant $\mathrm{SpO}_{2}$ fluctuations between and within multiple days and nights of patients with COPD. COPD patients are known to be a heterogeneous group [32,33], which leads to large differences in mean $\mathrm{SpO}_{2}$ values between patients. Day-to-day $\mathrm{SpO}_{2}$ fluctuations have not yet received a lot of attention in scientific literature and night-to-night fluctuations have not yet been studied over more than two nights in patients with COPD. Some studies examined intraday fluctuations, however, these studies only examined short-term $\mathrm{SpO}_{2}$ fluctuations (ie, measurements of one hour or less) in healthy individuals [29] or infants [26-28]. Our results showed that significant $\mathrm{SpO}_{2}$ fluctuations occurred between and within multiple days and nights for the included patients. This is often not taken into account in telemonitoring applications [12]. When using the current definition of nocturnal desaturation, 50\% $(10 / 20)$ of the included patients changed category between desaturator and nondesaturator over the course of 1 week.

Continuous $\mathrm{SpO}_{2}$ measurements with high amounts of valid data could be obtained during a 7-day monitoring period, except during MVPA (EE>3 MET). Nocturnal measurements were well-perceived by the patients, leading to the high compliance. These measurements only had a small amount of data that was considered invalid $(0.69 \%)$. This indicates that it is feasible to perform multi-night continuous $\mathrm{SpO}_{2}$ measurements. Most patients also performed daytime measurements during rest and LIPA (ie, 17 patients measured, on average, 7.6 hours per day).
These had a limited amount of invalid data (6.10\%). However, very few measurements were performed during MVPA (ie, 0.2 hours per day) and almost one-third of these measurements produced invalid data due to motion artifacts. Therefore, it might not be recommended to analyze continuous $\mathrm{SpO}_{2}$ measurements during periods of MVPA.

Two types of preprocessing steps for continuous $\mathrm{SpO}_{2}$ data have been reported: (1) removing deviating samples or (2) down-sampling the data. Deviating samples have been removed visually $[29,34]$ by using an unspecified data-cleaning algorithm [35] or by removing samples with a sample-to-sample deviation of more than 4\% [36] or 8\% [37]. The latter method, however, does not remove artifacts that last over longer periods of time (eg, see Figure 2B: around 17:00-18:00). Therefore, artifacts in this study were removed by removing blocks of deviating values instead of separate samples. After sample removal, Morillo et al excluded remaining artifacts by down-sampling the data from $8 \mathrm{~Hz}$ to $1 \mathrm{~Hz}$ [37]. However, autocorrelation analyses in our study showed that a sampling time of 1 sample per second still led to oversampling of the data (see Multimedia Appendix 2). $\mathrm{SpO}_{2}$ could be down-sampled to 1 sample per 20 seconds for improved artifact removal, without losing information about the $\mathrm{SpO}_{2}$ dynamics.

Mean nocturnal $\mathrm{SpO}_{2}$ was lower than mean daytime $\mathrm{SpO}_{2}$ in rest. By taking into account the $\mathrm{SpO}_{2}$ fluctuations over different days and nights, the results of this study generalize the findings of Soguel Schenkel et al, who only performed $\mathrm{SpO}_{2}$ measurements during a single day and night that were up to five days apart [38]. Other studies used awake measurements of 
$\mathrm{SpO}_{2}$ to predict nocturnal mean $\mathrm{SpO}_{2}$ or CT90. They used the first 15-30 minutes of the nocturnal measurements to calculate the mean awake $\mathrm{SpO}_{2}$ values [15,39-41]. For these studies, it should be noted that slightly lower $\mathrm{SpO}_{2}$ values were observed in the evening compared to the afternoon, on average a $0.8 \%$ difference, and awake $\mathrm{SpO}_{2}$ values depend on the time of day when the measurements are performed. It is unlikely that this difference is solely due to the specified oximeter accuracy of $\pm 2 \%$, as no consistent bias has been reported for the WristOx $\mathrm{x}_{2}$ when measuring over a longer time period.

The observed patient-to-patient, day-to-day, and intraday differences can have implications for telemonitoring applications that are based on daily $\mathrm{SpO}_{2}$ spot checks [12]. In several telemonitoring setups, alerts were raised when daily spot checks of $\mathrm{SpO}_{2}$ dropped below a generic threshold value (eg, $90 \%$ for all patients) [12]. However, the high patient-to-patient differences point out the shortcomings of these generic thresholds. Other telemonitoring setups used personalized thresholds, but these were still fixed on one specific threshold value for every patient [12]. For these setups, alerts can be merely a consequence of natural day-to-day and intraday fluctuations, instead of being triggered by the onset of an exacerbation. A recent paper better dealt with the day-to-day fluctuations by suggesting a day-to-day decrease of more than $4 \%$ to alert for exacerbations, however, a more thorough examination of this method is needed [42]. Intraday $\mathrm{SpO}_{2}$ values during rest ranged over $10.8 \%$ (SD 4.4) within one day. Moreover, statistical interpretation of weekly average of $\mathrm{SpO}_{2}$ $\mathrm{SD}(1.6 \%, \mathrm{SD} 0.6)$, which is a measure of intraday fluctuations, indicates that during $5 \%$ of the day in rest, $\mathrm{SpO}_{2}$ fluctuates more than $3.2 \%$ (ie, 2 times $\mathrm{SpO}_{2} \mathrm{SD}$ ) beyond the mean daytime-in-rest value. In comparison, previous studies only reported a $1 \%-2 \%$ decrease in $\mathrm{SpO}_{2}$ spot checks around exacerbation onset compared to stable periods [43-45]. These natural intraday fluctuations can thus easily result in false alerts for exacerbations. Patients with lower $\mathrm{SpO}_{2}$ values might experience a higher number of false alerts as, similar to healthy individuals [29], intraday fluctuations (ie, $\mathrm{SpO}_{2} \mathrm{SD}$ ) increased with decreasing mean $\mathrm{SpO}_{2}$. Altogether, personalized alerts based on intelligent algorithms will be necessary to cope with all of these natural fluctuations in daytime $\mathrm{SpO}_{2}$ in rest. Preferably, these alerts should be based on continuous measurements over longer time periods, in contrast to the currently used daily spot checks, to account for the identified $\mathrm{SpO}_{2}$ fluctuations and to exclude the potential effect of the $\pm 2 \%$ oximeter accuracy. In addition, the slightly lower $\mathrm{SpO}_{2}$ values in the evening compared to the afternoon show that the daily measurements should always be performed at the same moment of the day. A clearly defined measurement protocol, which is often not specified [12], can thus further attempt to limit the effect of natural $\mathrm{SpO}_{2}$ fluctuations in these telemonitoring applications.

The night-to-night differences of nocturnal mean $\mathrm{SpO}_{2}$ (ie, average weekly range of $2.0 \%$ over the different nights) led to highly varying CT90 values over the different nights of the measurement week (ie, average range of 28\%). These high variations in CT90 resulted in 50\% (10/20) of the included patients changing category between desaturator and nondesaturator, due to the fact that these occasional desaturators all had a mean $\mathrm{SpO}_{2}$ value around the threshold of $90 \%$. A similar finding has been reported by Lewis et al, who concluded that $35 \%$ of the included patients changed category over two consecutive nights of measurements [20]. Later studies then tackled this problem by performing measurements over two nights, categorizing a patient as a desaturator if desaturation occurred in at least one of both nights [5,17-19]. However, the results of this study indicate that even two nights are insufficient to make a consistent separation between desaturators and nondesaturators, as 6 out of 10 occasional desaturators $(60 \%)$ were desaturators or nondesaturators in the first two nights and only changed category afterward. Our results suggest that it might be impossible to consistently categorize COPD patients with a mean nocturnal $\mathrm{SpO}_{2}$ value around $90 \%$ as desaturator or nondesaturator. Based on measurements over multiple nights, these patients could thus be referred to as occasional desaturators. Further research is needed to assess the clinical relevance of identifying these three different nocturnal desaturation profiles.

Some limitations should be taken into consideration when interpreting the results of this study. The main limitation, as is often the case in similar studies, is the small sample size. Consequently, no comparison could be made between mild, moderate, and severe hypoxemic patients. Nevertheless, the increasing $\mathrm{SpO}_{2} \mathrm{SD}$ with decreasing mean $\mathrm{SpO}_{2}$ suggests an increase in $\mathrm{SpO}_{2}$ fluctuations for more hypoxemic patients. No control group was included because this study aimed to perform continuous $\mathrm{SpO}_{2}$ measurements in COPD patients for identification of $\mathrm{SpO}_{2}$ fluctuations that could affect $\mathrm{SpO}_{2}$ applications, rather than comparing $\mathrm{SpO}_{2}$ between COPD patients and healthy controls. In addition, daily home routines can greatly differ between patients and healthy controls [46], impeding a proper comparison. As shown in Multimedia Appendix 1, daytime measurements were only seldom performed during full days due to the impracticalities of wearing the finger clip during activities that require manual actions (eg, during morning routines). This, in combination with the high amount of invalid data during MVPA, suggests that continuous $\mathrm{SpO}_{2}$ measurements with a finger clip will have more profound limitations in a population that is more physically active than the target population of this study. Reliable wearable oximeters that do not require a finger clip could thus increase compliance. Moreover, the limited battery life and the inability of real-time data transmission of the used oximeter can further complicate the integration of continuous $\mathrm{SpO}_{2}$ measurements into practice. Technological advances are thus needed to allow for long-term, continuous monitoring of $\mathrm{SpO}_{2}$. This study, however, mainly intended to show the potential of prolonged continuous measurements to identify $\mathrm{SpO}_{2}$ fluctuations. Therefore, a certified wearable pulse oximeter with finger clip was preferred over more user-friendly, watch-type oximeters that have not yet been proven to be accurate. The resulting, more fragmented, daytime measurements were sufficient to identify large natural 
fluctuations occurring within one day (ie, $\mathrm{SpO}_{2}$ in rest ranged over $10.8 \%$ [SD 4.4] within one day), confirming the a priori posed hypothesis. The limited battery life was addressed by providing the patients with multiple sensors to cover the 7-day monitoring period.

This study was the first to use wearable finger pulse oximeters for prolonged continuous $\mathrm{SpO}_{2}$ measurements in COPD patients, as opposed to only performing spot checks or continuous measurements during one or two nights. These measurements showed that spot checks or one- or two-night measurements should be interpreted with caution, as the conclusions based on these measurements might change depending on the moment of the measurement. Measurements were performed at home during daily life routines of COPD patients, which provides a more natural $\mathrm{SpO}_{2}$ profile compared to supervised measurements. By adding actigraphy measurements, the necessary contextual information could be gathered for more accurate analyses of the continuous $\mathrm{SpO}_{2}$ measurements.

In conclusion, continuous $\mathrm{SpO}_{2}$ measurements with wearable pulse oximeters identified significant $\mathrm{SpO}_{2}$ fluctuations between and within multiple days and nights of patients with COPD. Continuous $\mathrm{SpO}_{2}$ measurements during the daily home routine of patients with COPD generally had high amounts of valid data, except for motion artifacts during MVPA. The continuous measurements showed that mean nocturnal $\mathrm{SpO}_{2}$ was lower than mean daytime $\mathrm{SpO}_{2}$ in rest, and significant $\mathrm{SpO}_{2}$ fluctuations occurred between and within multiple days and nights. The large fluctuations of daytime $\mathrm{SpO}_{2}$ in rest indicate that clear measurement protocols and personalized alerts, based on intelligent algorithms, will be needed to increase the performance of telemonitoring applications that make use of daily $\mathrm{SpO}_{2}$ spot checks. Lastly, it was shown that CT90 values can vary greatly from night to night in patients with a nocturnal mean $\mathrm{SpO}_{2}$ around $90 \%$, indicating that these patients cannot be consistently categorized as desaturators or nondesaturators. We recommend using wearable sensors for performing continuous $\mathrm{SpO}_{2}$ measurements over longer time periods to determine the clinical relevance of the identified $\mathrm{SpO}_{2}$ fluctuations.

\section{Acknowledgments}

This research is part of a PhD research project funded by the Flemish Institute for Technological Research (VITO), Mol, Belgium.

\section{Conflicts of Interest}

MAS discloses receipt of renumeration for consultancy and/or lectures from Boehringer Ingelheim, GlaxoSmithKline, and AstraZeneca outside the scope of this work. The authors have no other relevant affiliations or financial involvement with any organization or entity with a financial interest in or financial conflict with the subject matter or materials discussed in the manuscript, apart from those disclosed.

\section{Multimedia Appendix 1}

Visual overview of the continuous peripheral blood oxygen saturation $(\mathrm{SpO} 2)$ measurements that were included for analyses. [PDF File (Adobe PDF File), 32KB-Multimedia Appendix 1]

\section{Multimedia Appendix 2}

Autocorrelation analyses.

[PDF File (Adobe PDF File), 95KB-Multimedia Appendix 2]

\section{Multimedia Appendix 3}

Differences between mean peripheral blood oxygen saturation (SpO2) in the afternoon and in the evening. Grey dots indicate the differences within the same day for the same patient and orange dots indicate the average of these differences for every patient. The number of grey dots per patient indicate the amount of days with measurements in both the afternoon and evening.

[PDF File (Adobe PDF File), 22KB-Multimedia Appendix 3]

\section{References}

1. Global Strategy for the Diagnosis, Management, and Prevention of Chronic Obstructive Pulmonary Disease: 2019 Report. Fontana, WI: Global Initiative for Chronic Obstructive Lung Disease (GOLD); 2019. URL: https://goldcopd.org/wp-content/ uploads/2018/11/GOLD-2019-v1.7-FINAL-14Nov2018-WMS.pdf [accessed 2019-04-16] [WebCite Cache ID 77g4jvX6Y]

2. López-Campos JL, Tan W, Soriano JB. Global burden of COPD. Respirology 2016 Jan;21(1):14-23 [FREE Full text] [doi: 10.1111/resp.12660] [Medline: 26494423]

3. World Health Organization. 2019. Chronic obstructive pulmonary disease (COPD) URL: http://www.who.int/respiratory/ copd/en/ [accessed 2018-11-13] [WebCite Cache ID 73tsne72z] 
4. Casanova C, Hernández MC, Sánchez A, García-Talavera I, de Torres JP, Abreu J, et al. Twenty-four-hour ambulatory oximetry monitoring in COPD patients with moderate hypoxemia. Respir Care 2006 Dec;51(12):1416-1423 [FREE Full text] [Medline: 17134522$]$

5. Lacasse Y, Sériès F, Vujovic-Zotovic N, Goldstein R, Bourbeau J, Lecours R, et al. Evaluating nocturnal oxygen desaturation in COPD--revised. Respir Med 2011 Sep;105(9):1331-1337 [FREE Full text] [doi: 10.1016/j.rmed.2011.04.003] [Medline: 21561753]

6. Chaouat A, Weitzenblum E, Kessler R, Charpentier C, Ehrhart M, Levi-Valensi P, et al. Sleep-related O2 desaturation and daytime pulmonary haemodynamics in COPD patients with mild hypoxaemia. Eur Respir J 1997 Aug;10(8):1730-1735 [FREE Full text] [Medline: 9272911]

7. Weitzenblum E, Chaouat A. Sleep and chronic obstructive pulmonary disease. Sleep Med Rev 2004 Aug;8(4):281-294. [doi: 10.1016/j.smrv.2004.03.006] [Medline: 15233956]

8. Kent BD, Mitchell PD, McNicholas WT. Hypoxemia in patients with COPD: Cause, effects, and disease progression. Int J Chron Obstruct Pulmon Dis 2011;6:199-208 [FREE Full text] [doi: 10.2147/COPD.S10611] [Medline: 21660297]

9. Rodriguez-Roisin R. Toward a consensus definition for COPD exacerbations. Chest 2000 May;117(5 Suppl 2):398S-401S. [doi: 10.1378/chest.117.5 suppl 2.398s] [Medline: 10843984]

10. Kelly AM, McAlpine R, Kyle E. How accurate are pulse oximeters in patients with acute exacerbations of chronic obstructive airways disease? Respir Med 2001 May;95(5):336-340 [FREE Full text] [doi: 10.1053/rmed.2001.1046] [Medline: 11392573]

11. Garcia-Gutierrez S, Unzurrunzaga A, Arostegui I, Quintana JM, Pulido E, Gallardo MS, IRYSS-COPD group. The use of pulse oximetry to determine hypoxemia in acute exacerbations of COPD. COPD 2015;12(6):613-620. [doi: 10.3109/15412555.2014.995291] [Medline: 25774875]

12. Buekers J, De Boever P, Vaes AW, Aerts J, Wouters EFM, Spruit MA, et al. Oxygen saturation measurements in telemonitoring of patients with COPD: A systematic review. Expert Rev Respir Med 2018 Dec;12(2):113-123. [doi: 10.1080/17476348.2018.1417842] [Medline: 29241369]

13. Toraldo DM, Nicolardi G, De Nuccio F, Lorenzo R, Ambrosino N. Pattern of variables describing desaturator COPD patients, as revealed by cluster analysis. Chest 2005 Dec;128(6):3828-3837. [doi: 10.1378/chest.128.6.3828] [Medline: $\underline{16354851]}$

14. Scott AS, Baltzman MA, Chan R, Wolkove N. Oxygen desaturation during a 6 min walk test is a sign of nocturnal hypoxemia. Can Respir J 2011;18(6):333-337 [FREE Full text] [doi: 10.1155/2011/242636] [Medline: 22187689]

15. Levi-Valensi P, Weitzenblum E, Rida Z, Aubry P, Braghiroli A, Donner C, et al. Sleep-related oxygen desaturation and daytime pulmonary haemodynamics in COPD patients. Eur Respir J 1992 Mar;5(3):301-307. [Medline: 1572442]

16. Iliaz S, Cagatay T, Bingol Z, Okumus G, Iliaz R, Kuran G, et al. Does the 6-minute walk test predict nocturnal oxygen desaturation in patients with moderate to severe COPD? Chron Respir Dis 2015 Feb;12(1):61-68. [doi: 10.1177/1479972314562406] [Medline: 25480424]

17. Nisbet M, Eaton T, Lewis C, Fergusson W, Kolbe J. Overnight prescription of oxygen in long term oxygen therapy: Time to reconsider the guidelines? Thorax 2006 Sep;61(9):779-782 [FREE Full text] [doi: 10.1136/thx.2005.056119] [Medline: $\underline{16769716]}$

18. Lacasse Y, Bernard S, Sériès F, Nguyen VH, Bourbeau J, Aaron S, International Nocturnal Oxygen (INOX) Research Group. Multi-center, randomized, placebo-controlled trial of nocturnal oxygen therapy in chronic obstructive pulmonary disease: A study protocol for the INOX trial. BMC Pulm Med 2017 Dec 09;17(1):8. [doi: 10.1186/s12890-016-0343-9] [Medline: 28069009]

19. Lewis CA, Fergusson W, Eaton T, Zeng I, Kolbe J. Isolated nocturnal desaturation in COPD: Prevalence and impact on quality of life and sleep. Thorax 2009 Feb;64(2):133-138. [doi: 10.1136/thx.2007.088930] [Medline: 18390630]

20. Lewis CA, Eaton TE, Fergusson W, Whyte KF, Garrett JE, Kolbe J. Home overnight pulse oximetry in patients with COPD: More than one recording may be needed. Chest 2003 Apr;123(4):1127-1133. [doi: 10.1378/chest.123.4.1127] [Medline: $\underline{12684303]}$

21. Spruit MA, Vanderhoven-Augustin I, Janssen PP, Wouters EFM. Integration of pulmonary rehabilitation in COPD. Lancet 2008 Jan 05;371(9606):12-13. [doi: 10.1016/S0140-6736(08)60048-3] [Medline: 18177760]

22. Nonin Medical. WristOx2 ® Model 3150 USB: Wrist-worn pulse oximeter URL: https://www.nonin.com/wp-content/ uploads/2018/09/Spec-Sheet-WristOx2-Model-3150-USB.pdf [accessed 2019-04-04] [WebCite Cache ID 77Ni6Sp3k]

23. Van Remoortel H, Raste Y, Louvaris Z, Giavedoni S, Burtin C, Langer D, PROactive consortium. Validity of six activity monitors in chronic obstructive pulmonary disease: A comparison with indirect calorimetry. PLoS One 2012;7(6):e39198 [FREE Full text] [doi: 10.1371/journal.pone.0039198] [Medline: 22745715]

24. Hill K, Dolmage TE, Woon L, Goldstein R, Brooks D. Measurement properties of the SenseWear armband in adults with chronic obstructive pulmonary disease. Thorax 2010 Jun;65(6):486-491. [doi: 10.1136/thx.2009.128702] [Medline: 20522844]

25. Sharif MM, Bahammam AS. Sleep estimation using BodyMedia's SenseWear ${ }^{\mathrm{TM}}$ armband in patients with obstructive sleep apnea. Ann Thorac Med 2013 Jan;8(1):53-57 [FREE Full text] [doi: 10.4103/1817-1737.105720] [Medline: 23440703] 
26. de Jesus JA, Tristao RM, Storm H, da Rocha AF, Campos D. Heart rate, oxygen saturation, and skin conductance: A comparison study of acute pain in Brazilian newborns. Conf Proc IEEE Eng Med Biol Soc 2011;2011:1875-1879. [doi: 10.1109/IEMBS.2011.6090532] [Medline: 22254696]

27. de Oliveira MV, de Jesus JA, Tristao RM. Psychophysical parameters of a multidimensional pain scale in newborns. Physiol Meas 2012 Jan;33(1):39-49. [doi: 10.1088/0967-3334/33/1/39] [Medline: 22155897]

28. Garde A, Zhou G, Raihana S, Dunsmuir D, Karlen W, Dekhordi P, et al. Respiratory rate and pulse oximetry derived information as predictors of hospital admission in young children in Bangladesh: A prospective observational study. BMJ Open 2016 Dec 17;6(8):e011094 [FREE Full text] [doi: 10.1136/bmjopen-2016-011094] [Medline: 27534987]

29. Bhogal AS, Mani AR. Pattern analysis of oxygen saturation variability in healthy individuals: Entropy of pulse oximetry signals carries information about mean oxygen saturation. Front Physiol 2017;8:555 [FREE Full text] [doi: 10.3389/fphys.2017.00555] [Medline: 28824451]

30. Jupyter. URL: https://jupyter.org/ [accessed 2019-05-21] [WebCite Cache ID 78XYVWOKv]

31. Python. URL: https://www.python.org/ [accessed 2019-05-21] [WebCite Cache ID 78XYTIZtV]

32. Sillen MJ, Franssen FM, Delbressine JM, Uszko-Lencer NH, Vanfleteren LE, Rutten EP, et al. Heterogeneity in clinical characteristics and co-morbidities in dyspneic individuals with COPD GOLD D: Findings of the DICES trial. Respir Med 2013 Aug;107(8):1186-1194 [FREE Full text] [doi: 10.1016/j.rmed.2013.04.020] [Medline: 23706780]

33. Agusti A, Calverley PM, Celli B, Coxson HO, Edwards LD, Lomas DA, Evaluation of COPD Longitudinally to Identify Predictive Surrogate Endpoints (ECLIPSE) investigators. Characterisation of COPD heterogeneity in the ECLIPSE cohort. Respir Res 2010 Sep 10;11:122 [FREE Full text] [doi: 10.1186/1465-9921-11-122] [Medline: 20831787]

34. Fussell KM, Ayo DS, Branca P, Rogers JT, Rodriguez M, Light RW. Assessing need for long-term oxygen therapy: A comparison of conventional evaluation and measures of ambulatory oximetry monitoring. Respir Care 2003 Feb;48(2):115-119 [FREE Full text] [Medline: 12556251]

35. Álvarez D, Hornero R, Marcos JV, Del Campo F. Feature selection from nocturnal oximetry using genetic algorithms to assist in obstructive sleep apnoea diagnosis. Med Eng Phys 2012 Oct;34(8):1049-1057. [doi: 10.1016/j.medengphy.2011.11.009] [Medline: 22154238]

36. Marcos JV, Hornero R, Nabney IT, Álvarez D, Gutiérrez-Tobal GC, del Campo F. Regularity analysis of nocturnal oximetry recordings to assist in the diagnosis of sleep apnoea syndrome. Med Eng Phys 2016 Mar;38(3):216-224. [doi: 10.1016/j.medengphy.2015.11.010] [Medline: 26719242]

37. Morillo DS, Gross N. Probabilistic neural network approach for the detection of SAHS from overnight pulse oximetry. Med Biol Eng Comput 2013 Mar;51(3):305-315. [doi: 10.1007/s11517-012-0995-4] [Medline: 23160897]

38. Soguel Schenkel N, Burdet L, de Muralt B, Fitting JW. Oxygen saturation during daily activities in chronic obstructive pulmonary disease. Eur Respir J 1996 Dec;9(12):2584-2589 [FREE Full text] [Medline: 8980973]

39. Mulloy E, Fitzpatrick M, Bourke S, O'Regan A, McNicholas WT. Oxygen desaturation during sleep and exercise in patients with severe chronic obstructive pulmonary disease. Respir Med 1995 Mar;89(3):193-198 [FREE Full text] [Medline: 7746912]

40. Heijdra YF, Dekhuijzen PN, van Herwaarden CL, Folgering HT. Nocturnal saturation and respiratory muscle function in patients with chronic obstructive pulmonary disease. Thorax 1995 Jun;50(6):610-612 [FREE Full text] [doi: 10.1136/thx.50.6.610] [Medline: $\underline{7638800]}$

41. Mohsenin V, Guffanti EE, Hilbert J, Ferranti R. Daytime oxygen saturation does not predict nocturnal oxygen desaturation in patients with chronic obstructive pulmonary disease. Arch Phys Med Rehabil 1994 Mar;75(3):285-289. [Medline: $\underline{8129580]}$

42. Miłkowska-Dymanowska J, Białas AJ, Obrębski W, Górski P, Piotrowski WJ. A pilot study of daily telemonitoring to predict acute exacerbation in chronic obstructive pulmonary disease. Int J Med Inform 2018 Dec;116:46-51 [FREE Full text] [doi: 10.1016/j.ijmedinf.2018.04.013] [Medline: 29887234]

43. Martín-Lesende I, Orruño E, Bilbao A, Vergara I, Cairo MC, Bayón JC, et al. Impact of telemonitoring home care patients with heart failure or chronic lung disease from primary care on healthcare resource use (the TELBIL study randomised controlled trial). BMC Health Serv Res 2013 Mar 28;13:118 [FREE Full text] [doi: 10.1186/1472-6963-13-118] [Medline: 23537332]

44. Burton C, Pinnock H, McKinstry B. Changes in telemonitored physiological variables and symptoms prior to exacerbations of chronic obstructive pulmonary disease. J Telemed Telecare 2015 Jan;21(1):29-36. [doi: 10.1177/1357633X14562733] [Medline: 25475218]

45. Shah SA, Velardo C, Farmer A, Tarassenko L. Exacerbations in chronic obstructive pulmonary disease: Identification and prediction using a digital health system. J Med Internet Res 2017 Dec 07;19(3):e69 [FREE Full text] [doi: 10.2196/jmir.7207] [Medline: 28270380]

46. Troosters T, Sciurba F, Battaglia S, Langer D, Valluri SR, Martino L, et al. Physical inactivity in patients with COPD, a controlled multi-center pilot-study. Respir Med $2010 \mathrm{Jul}$;104(7):1005-1011 [FREE Full text] [doi:

10.1016/j.rmed.2010.01.012] [Medline: 20167463] 


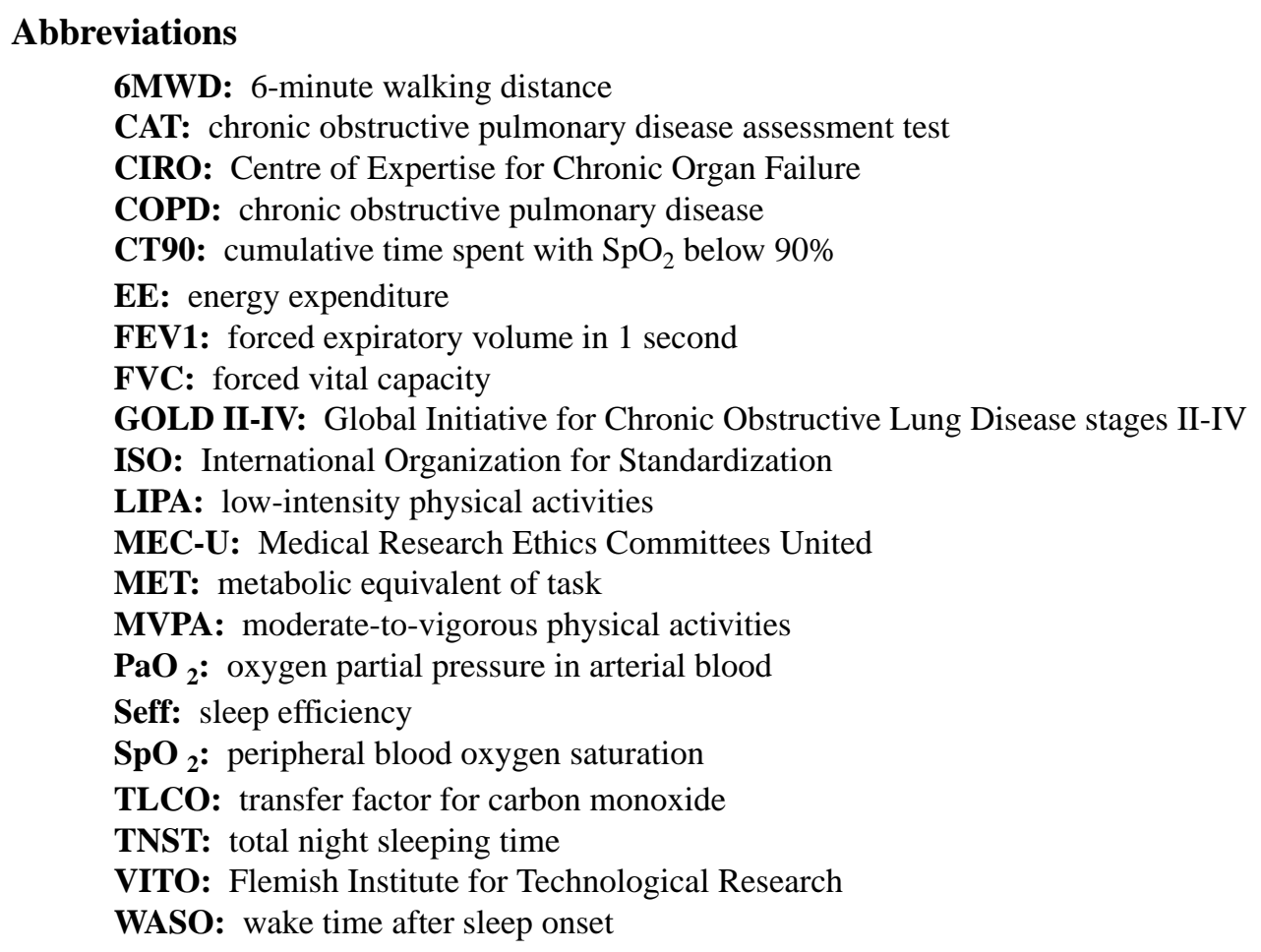

Edited by G Eysenbach; submitted 21.11.18; peer-reviewed by N Hernandes, YR Park, A Farmer, D Dunsmuir; comments to author 31.03.19; revised version received 16.04.19; accepted 27.04.19; published 06.06.19

Please cite as:

Buekers J, Theunis J, De Boever P, Vaes AW, Koopman M, Janssen EVM, Wouters EFM, Spruit MA, Aerts JM

Wearable Finger Pulse Oximetry for Continuous Oxygen Saturation Measurements During Daily Home Routines of Patients With Chronic Obstructive Pulmonary Disease (COPD) Over One Week: Observational Study

JMIR Mhealth Uhealth 2019;7(6):e12866

URL: https://mhealth.jmir.org/2019/6/e12866/

doi: $\underline{10.2196 / 12866}$

PMID: 31199331

CJoren Buekers, Jan Theunis, Patrick De Boever, Anouk W Vaes, Maud Koopman, Eefje VM Janssen, Emiel FM Wouters, Martijn A Spruit, Jean-Marie Aerts. Originally published in JMIR Mhealth and Uhealth (http://mhealth.jmir.org), 06.06.2019. This is an open-access article distributed under the terms of the Creative Commons Attribution License (https://creativecommons.org/licenses/by/4.0/), which permits unrestricted use, distribution, and reproduction in any medium, provided the original work, first published in JMIR mhealth and uhealth, is properly cited. The complete bibliographic information, a link to the original publication on http://mhealth.jmir.org/, as well as this copyright and license information must be included. 